\title{
Dimensões da gestão do cuidado na prática do enfermeiro na atenção primária: revisão integrativa
}

\author{
Dimensions of care management in primary care nurses' practice: integrative review
}

Dimensiones de la gestión de la atención en la práctica de enfermeros de atención primaria: revisión integradora

\author{
Fernanda Karla Metelskil @ ; Thaís Fávero Alves ${ }^{\circledR} \odot$, Rosiane da Rosal $\odot$, \\ José Luís Guedes dos Santos' ${ }^{\circledR}$, Selma Regina Andradel ${ }^{\circledR}$
}

'Universidade Federal de Santa Catarina, Florianópolis, SC, Brasil

\begin{abstract}
RESUMO
Objetivo: evidenciar as dimensões da gestão do cuidado na prática do enfermeiro na Atenção Primária à Saúde. Método: revisão integrativa nas bases de dados: SCOPUS, LILACS, BDENF, MEDLINE, CINAHL, e Web of Science. Foram incluídos artigos originais disponíveis on-line na íntegra, publicados em português, inglês ou espanhol, entre 2012 e 2018. Utilizou-se a análise de conteúdo considerando as seis dimensões da gestão do cuidado: individual, familiar, profissional, organizacional, sistêmica e societária. Resultados: entre os 24 estudos analisados, predominaram estudos qualitativos e nacionais. Todas as dimensões estão presentes na prática do enfermeiro. Conclusão: sobressaiu-se a dimensão organizacional, ressaltando a atuação do enfermeiro como gestor, gerente ou coordenador, por meio do exercício da liderança, planejamento e desenvolvimento de atividades gerenciais integradas à assistência. Pontua-se a importância da busca do enfermeiro pelo desenvolvimento contínuo de competências para a gestão do cuidado.

Descritores: Gestão em Saúde; Atenção Primária à Saúde; Enfermagem; Papel do Profissional de Enfermagem; Organização e Administração.
\end{abstract}

\section{ABSTRACT}

Objective: to highlight dimensions of care management in the practice of Primary Health Care nurses. Method: this integrative review searched the SCOPUS, LILACS, BDENF, MEDLINE, CINAHL, and Web of Science databases for full-version original articles published in Portuguese, English or Spanish between 2012 and 2018. Content analysis was used considering the six dimensions - individual, family, professional, organizational, systemic and corporate - of care management. Results: the 24 studies analyzed were predominantly Brazilian and qualitative. All the dimensions were present in the nurses' practice. Conclusion: the organizational dimension was most prominent, underlining the nurse's role as manager or coordinator, exercising leadership, planning and managerial activities integrated with care. Attention is drawn to the importance of nurse's engaging in continuous development of care management skills.

Descriptors: Health Management; Primary Health Care; Nursing; Nurse's Role; Organization and Administration.

\section{RESUMEN}

Objetivo: destacar las dimensiones de la gestión asistencial en la práctica del enfermero de Atención Primaria de Salud. Método: esta revisión integradora buscó en las bases de datos SCOPUS, LILACS, BDENF, MEDLINE, CINAHL y Web of Science artículos originales en versión completa publicados en portugués, inglés o español entre 2012 y 2018. Se utilizó el análisis de contenido considerando las seis dimensiones: individual , familiar, profesional, organizativa, sistémica y corporativa - de gestión asistencial. Resultados: los 24 estudios analizados fueron predominantemente brasileños y cualitativos. Todas las dimensiones estuvieron presentes en la práctica de las enfermeras. Conclusión: la dimensión organizacional fue la más destacada, destacando el rol de la enfermera como gerente o coordinadora, ejerciendo el liderazgo, planificación y actividades gerenciales integradas con el cuidado. Se llama la atención sobre la importancia de que la enfermera participe en el desarrollo continuo de las habilidades de gestión de la atención.

Descriptores: Gestión en Salud; Atención Primaria de Salud; Enfermería; Rol de la Enfermera; Organización y Administración.

\section{INTRODUÇÃO}

O trabalho do enfermeiro na Atenção Primária à Saúde (APS) está focado em ações gerenciais e assistenciais, por meio da produção do cuidado, do gerenciamento do serviço de saúde e da gestão da equipe de enfermagem ${ }^{1-6}$. Essas atividades ocorrem de forma integrada e podem ser definidas como gestão do cuidado, termo que tem sido utilizado para se referir à articulação entre as esferas gerencial e assistencial, como, por exemplo, no trabalho do enfermeiro ${ }^{1,4-}$ ${ }^{6}$. Do ponto de vista ético-legal da profissão, a gestão do cuidado está relacionada às atividades privativas do enfermeiro em relação a planejar, executar, coordenar, supervisionar e avaliar a assistência de enfermagem ${ }^{7}$.

Na APS, por meio da gestão do cuidado, o enfermeiro contribui para a qualificação da assistência e para o desenvolvimento de um cuidado integral ${ }^{1}$. Nesse sentido, os enfermeiros têm uma posição importante atuando tanto

Agradecimentos à Coordenação de Aperfeiçoamento de Pessoal de Nível Superior (CAPES) - PROEX 0541/2017.

Autora correspondente: Fernanda Karla Metelski. E-mail: fernanda.metelski@udesc.br

Editora responsável: Cintia Fassarella 
na articulação e integração de ações, quanto no trabalho em equipe para a resolução dos problemas de saúde dos pacientes e coletividades, o que requer ações pautadas na racionalidade técnico-científica, sem desconsiderar a imprevisibilidade das interações humanas ${ }^{8,9}$.

A gestão do cuidado emprega tecnologias em saúde, direcionadas às necessidades singulares de cada pessoa para os diferentes momentos de sua vida, em busca de promover o seu bem-estar, desenvolver sua autonomia, obter uma vida produtiva e feliz. Envolve o saber-fazer em seis dimensões inter-relacionadas: individual, familiar, profissional, organizacional, sistêmica e societária帛10.

A dimensão individual remete ao cuidado de si, autonomia, e fazer escolhas, sendo capaz de conduzir a própria vida, e produzindo novos e plenos modos de viver. A dimensão familiar contempla a família, vizinhos e amigos, e envolve o apoio, a proximidade e o mundo da vida. Na dimensão profissional acontece o encontro entre o profissional e o usuário, concebendo a competência técnica, postura ética, e construção de vínculos. A dimensão organizacional remete à divisão técnica e social do trabalho, à organização do processo de trabalho em equipe e à função gerencial propriamente dita. A dimensão sistêmica considera as conexões formais, regulares e regulamentadas entre os serviços de saúde, compondo linhas de cuidado, e ocupando-se também do financiamento em saúde. Na dimensão societária, a sociedade civil e Estado disputam ou colaboram em busca de uma vida melhor ${ }^{10}$

Estudos anteriores sobre gestão do cuidado têm focado principalmente o contexto hospitalar e discussões teórico-

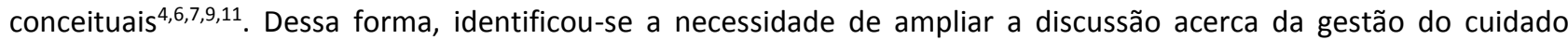
considerando as suas múltiplas dimensões ${ }^{10}$ na prática profissional do enfermeiro na APS. Assim, este estudo teve como objetivo evidenciar as dimensões da gestão do cuidado na prática do enfermeiro na APS.

\section{MÉTODO}

Adotou-se a revisão integrativa, com seis etapas: (1) definição de questão de pesquisa; (2) estabelecimento dos critérios de seleção dos estudos; (3) representação dos estudos selecionados em tabelas; (4) avaliação dos estudos e análise crítica dos resultados; (5) discussão e interpretação dos resultados e (6) reportar a revisão com as evidências encontradas ${ }^{12}$.

A partir do panorama apresentado anteriormente, definiu-se como questão de pesquisa: De que forma as dimensões da gestão do cuidado estão presentes na prática do enfermeiro na APS?

A estratégia de identificação e seleção dos estudos foi a busca de publicações indexadas em seis bases de dados: SCOPUS; Literatura Latino Americana em Ciências da Saúde (LILACS); Base de Dados Bibliográficos Especializada na Área de Enfermagem (BDENF); Medical Literature Analysis and Retrieval System Online (MEDLINE); Cumulative Index to Nursing and Allied Health Literature (CINAHL); e Web of Science. Foram utilizados os seguintes descritores com o operador booleano "and", e suas combinações na língua portuguesa, espanhola e inglesa: "Gestão em Saúde"; “Atenção Primária à Saúde"; e "Enfermagem".

A coleta de dados foi realizada em novembro de 2019. Os critérios de inclusão definidos para a seleção dos estudos foram artigos originais completos, de livre acesso e oriundos de pesquisas que abordassem a temática em foco no recorte histórico de 2012 a 2018. O ano de 2012 foi estabelecido como recorte temporal inicial devido à vigência da segunda versão da Política Nacional de Atenção Básica (PNAB) no Brasil e suas reedições, cujo conteúdo destaca-se como marco histórico para a instituição da Estratégia de Saúde da Família (ESF) como modelo prioritário de atenção à saúde $^{13}$. A busca considerou artigos publicados até 2018 para incluir somente anos completos na revisão. Artigos que não atenderam ao objetivo da pesquisa foram excluídos do estudo.

O filtro inicial nas bases de dados obteve 92 artigos, sendo: 32 artigos na Web of Science; 23 na LILACS; 18 na MEDLINE; nove na CINAHL; sete na BDENF; e três na SCOPUS. A análise de conteúdo mediante pré-análise, exploração do material e tratamento dos resultados ${ }^{14}$ foi utilizada para identificar os conceitos-chave relacionados às dimensões da gestão do cuidado ${ }^{9-10}$.

Na pré-análise, procedeu-se a leitura do título, resumo, e leitura flutuante do artigo, buscando identificar as dimensões da gestão do cuidado presentes no texto. Nesta etapa, dos 92 artigos supracitados, 65 artigos foram excluídos, sendo pré-selecionados 27 artigos, os quais após a leitura na íntegra, três foram excluídos por não atenderem ao objetivo da revisão (Figura 1). A próxima etapa foi o tratamento dos resultados por meio da leitura em profundidade dos artigos, possibilitando a interpretação dos achados e a confirmação ou não das categorias previamente elencadas ${ }^{10}$. O material foi organizado com o auxílio dos softwares Excel ${ }^{\circledR} 2013$ e ATLAS.ti $^{\circledR}$ versão 8. 


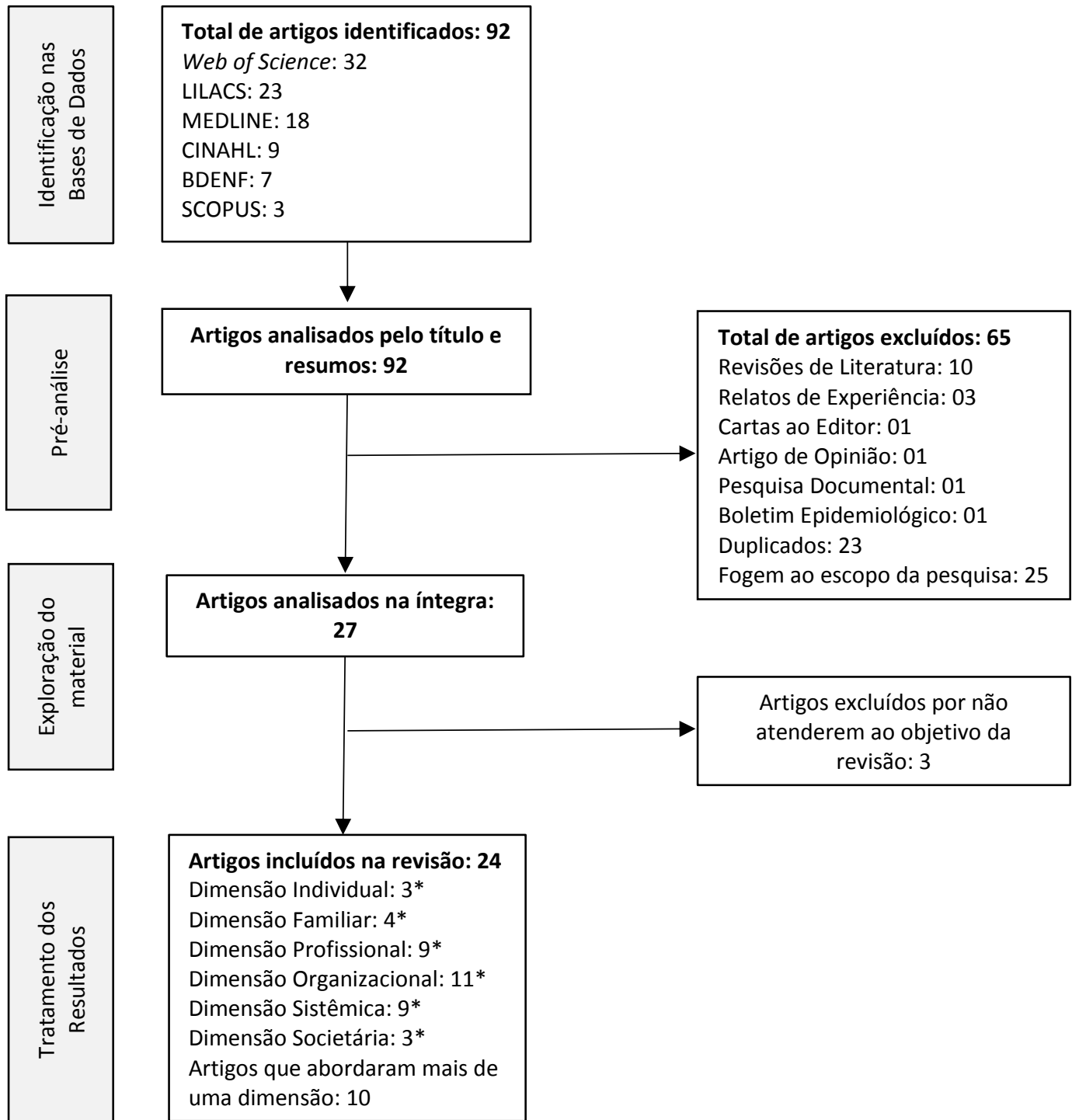

Nota: *O total de artigos para esta dimensão incluiu alguns que abordaram mais de uma dimensão.

FIGURA 1: Fluxograma da síntese das etapas de seleção e análise. Florianópolis, Santa Catarina, Brasil, 2019.

\section{RESULTADOS}

No total, 24 artigos $^{15-38}$ compuseram a amostra final, com destaque para os anos 2016 e 2013, com nove e cinco artigos, respectivamente (Figura 2).

A maioria dos periódicos apresentaram apenas uma publicação, com exceção do Population Heath Management, Revista da Escola de Enfermagem da USP, Revista Enfermagem UERJ, e Revista Gaúcha de Enfermagem, com duas publicações cada.

O local do estudo dos artigos nacionais segundo o Estado da Federação foram: Rio Grande do Sul com quatro artigos; Ceará e São Paulo três cada um; Goiás, Paraíba, e Minas Gerais obtiveram dois; e Paraná com uma publicação. Os estudos internacionais originaram-se da Finlândia, Inglaterra, Bósnia e Herzegovina, Albânia, Ruanda, e dois dos Estados Unidos.

De modo geral, os estudos analisaram ações desenvolvidas por enfermeiros em conjunto com outros participantes, sendo que: seis $(25 \%)$ envolveram a equipe multiprofissional; seis $(25 \%)$ contemplaram exclusivamente os enfermeiros; quatro (17\%) foram realizados com pacientes; dois ( $8 \%$ ) com enfermeiros e pacientes; dois (8\%) com profissionais de enfermagem no geral; dois (8\%) com enfermeiros e médicos; um (4\%) com enfermeiros e gestores; e um $(4 \%)$ com gerentes. Os estudos de natureza qualitativa totalizaram $15(63 \%)$, seguido dos quantitativos com oito (33\%), e abordagem mista com um (4\%). 


\begin{tabular}{|c|c|c|}
\hline Ano & Revista & Título \\
\hline \multirow[t]{3}{*}{2012} & $\begin{array}{l}\text { Revista da Rede de Enfermagem } \\
\text { do Nordeste }\end{array}$ & Trabalho do Apoiador Matricial na estratégia de saúde da família ${ }^{15}$ \\
\hline & $\begin{array}{l}\text { Revista Gerencia y Políticas de } \\
\text { Salud }\end{array}$ & $\begin{array}{l}\text { Análise do processo de trabalho dos gerentes no território da Estratégia Saúde da } \\
\text { Família16 }\end{array}$ \\
\hline & Revista Enfermagem UERJ & Cuidado ao adolescente: contribuições para a enfermagem ${ }^{17}$ \\
\hline \multirow[t]{5}{*}{2013} & BMJ & $\begin{array}{l}\text { Implementation of self management support for long term conditions in routine } \\
\text { primary care settings: cluster randomized controlled trial18 }\end{array}$ \\
\hline & Population Health Management & $\begin{array}{l}\text { Effects of Guided Care on Providers' Satisfaction with Care: a Three-Year Matched- } \\
\text { Pair Cluster-Randomized Trial }{ }^{19}\end{array}$ \\
\hline & Revista Gaúcha de Enfermagem & $\begin{array}{l}\text { Trabalho do apoiador matricial: dificuldades no âmbito da atenção básica em } \\
\text { saúde }\end{array}$ \\
\hline & Revista Gaúcha de Enfermagem & $\begin{array}{l}\text { Coordenação de unidades de saúde da família por enfermeiros: desafios e } \\
\text { potencialidades }{ }^{21}\end{array}$ \\
\hline & Revista Enfermagem UERJ & Comissão de saúde mental: estratégias na busca de espaços na atenção básica ${ }^{22}$ \\
\hline 2014 & $\begin{array}{l}\text { Revista da Escola de } \\
\text { Enfermagem da USP }\end{array}$ & $\begin{array}{l}\text { Supervisão dos Agentes Comunitários de Saúde na Estratégia Saúde da Família: a } \\
\text { ótica dos enfermeiros }{ }^{23}\end{array}$ \\
\hline 2015 & $\begin{array}{l}\text { Revista Brasileira de } \\
\text { Enfermagem }\end{array}$ & $\begin{array}{l}\text { Percepção dos enfermeiros gestores da atenção primária sobre o Processo de } \\
\text { Enfermagem }(P E)^{24}\end{array}$ \\
\hline \multirow[t]{9}{*}{2016} & $\begin{array}{l}\text { International Journal of } \\
\text { Circumpolar Health }\end{array}$ & Adherence to health regimens among frequent attenders of Finnish healthcare ${ }^{25}$ \\
\hline & $\begin{array}{l}\text { Revista da Escola de } \\
\text { Enfermagem da USP }\end{array}$ & $\begin{array}{l}\text { Formação de médicos e enfermeiros da estratégia Saúde da Família no aspecto da } \\
\text { saúde do trabalhador }{ }^{26}\end{array}$ \\
\hline & International Nursing Review & $\begin{array}{l}\text { Improving the safety and quality of nursing care through standardized operating } \\
\text { procedures in Bosnia and Herzegovina }{ }^{27}\end{array}$ \\
\hline & Journal of Nursing Scholarship & Provider Perspectives on Safety in Primary Care in Albania ${ }^{28}$ \\
\hline & $\begin{array}{l}\text { Revista de Enfermagem da UFPE } \\
\text { on line }\end{array}$ & $\begin{array}{l}\text { A coordenação da assistência no controle da tuberculose na visão da equipe de } \\
\text { enfermagem } 29\end{array}$ \\
\hline & Revista de APS & $\begin{array}{l}\text { Gestão da atenção a usuários com dependência de cuidados por sequelas de } \\
\text { acidente vascular cerebral }{ }^{30}\end{array}$ \\
\hline & Ciência, Cuidado \& Saúde & Gerenciamento em saúde: o olhar de trabalhadores da saúde da família rural ${ }^{31}$ \\
\hline & Population Health Management & $\begin{array}{l}\text { Collaborative Depression Care in a Safety Net Medical Home: Facilitators and } \\
\text { Barriers to Quality Improvement }{ }^{32}\end{array}$ \\
\hline & Texto \& Contexto Enfermagem & $\begin{array}{l}\text { Gestão do cuidado da tuberculose: integrando um hospital de ensino à atenção } \\
\text { primária à saúde }\end{array}$ \\
\hline \multirow[t]{2}{*}{2017} & $\begin{array}{l}\text { Investigación y Educación en } \\
\text { Enfermería }\end{array}$ & Perfil de diagnósticos de enfermagem em pessoas hipertensas e diabéticas ${ }^{34}$ \\
\hline & $\begin{array}{l}\text { Revista de Enfermagem da UFPE } \\
\text { on line }\end{array}$ & Gestão do trabalho em unidades básicas de saúde ${ }^{35}$ \\
\hline \multirow[t]{3}{*}{2018} & Enfermagem em Foco & $\begin{array}{l}\text { Desafios da gestão do cuidado na atenção básica: perspectiva da equipe de } \\
\text { enfermagem }{ }^{36}\end{array}$ \\
\hline & BMC Health Services Research & Process evaluation of a National Primary Eye Care Programme in Rwanda ${ }^{37}$ \\
\hline & Ciência \& Saúde Coletiva & $\begin{array}{l}\text { Notificação da violência infantil, fluxos de atenção e processo de trabalho dos } \\
\text { profissionais da Atenção Primária em Saúde }\end{array}$ \\
\hline
\end{tabular}

FIGURA 2: Caracterização dos estudos da amostra no período de 2012 a 2018. Florianópolis, Santa Catarina, Brasil, 2019.

A Figura 3 demonstra a síntese dos principais achados dessa revisão. Os achados foram agrupados de acordo com as similaridades nas dimensões da gestão do cuidado: individual; familiar; profissional; organizacional; sistêmica; societária. 


\begin{tabular}{|c|c|}
\hline $\begin{array}{l}\text { Dimensão da Gestão do } \\
\text { Cuidado } \\
\text { / totalidade de } \\
\text { referências incluídas. }\end{array}$ & Principais resultados envolvendo a prática do Enfermeiro na Atenção Primária à Saúde \\
\hline $\begin{array}{l}\text { Individual } \\
/ 18,25,32\end{array}$ & $\begin{array}{l}\text { - auto manejo }{ }^{18,32} \\
\text { - autocuidado } 25 \\
\text { - escolha de estilos de vida }{ }^{32}\end{array}$ \\
\hline $\begin{array}{l}\text { Familiar } \\
/ 19,25,30,32 .\end{array}$ & $\begin{array}{l}\text { - apoio para cuidadores familiares }{ }^{19} \\
\text { - suporte de parentes e amigos } \\
\text { - terapia de família } \\
\text { - }{ }^{32}\end{array}$ \\
\hline $\begin{array}{l}\text { Profissional } \\
/ 19,20,24,26,27,28 \\
31,34,35\end{array}$ & $\begin{array}{l}\text { - integralidade no cuidado } \\
\text { - consulta de enfermagem e processo de enfermagem }{ }^{24,34} \\
\text { - prática baseada em evidências e plano de } \text { cuidados }^{19,34} \\
\text { - procedimentos padronizados }{ }^{27} \\
\text { - desenvolver competências profissionais } 26-27,35 \\
\text { - carga de trabalho } \\
\text { - vínculo entre enfermeiros e pacientes e preocupação com a prestação de cuidados }{ }^{27} \\
\text { - discussão de temas frente a opiniões discordantes }{ }^{28} \\
\text { - relação entre enfermeiro e equipe de saúde }{ }^{20,31,35}\end{array}$ \\
\hline $\begin{array}{l}\text { Organizacional } \\
/ 15,16,17,19,20,21 \\
24,29,30,31,35\end{array}$ & 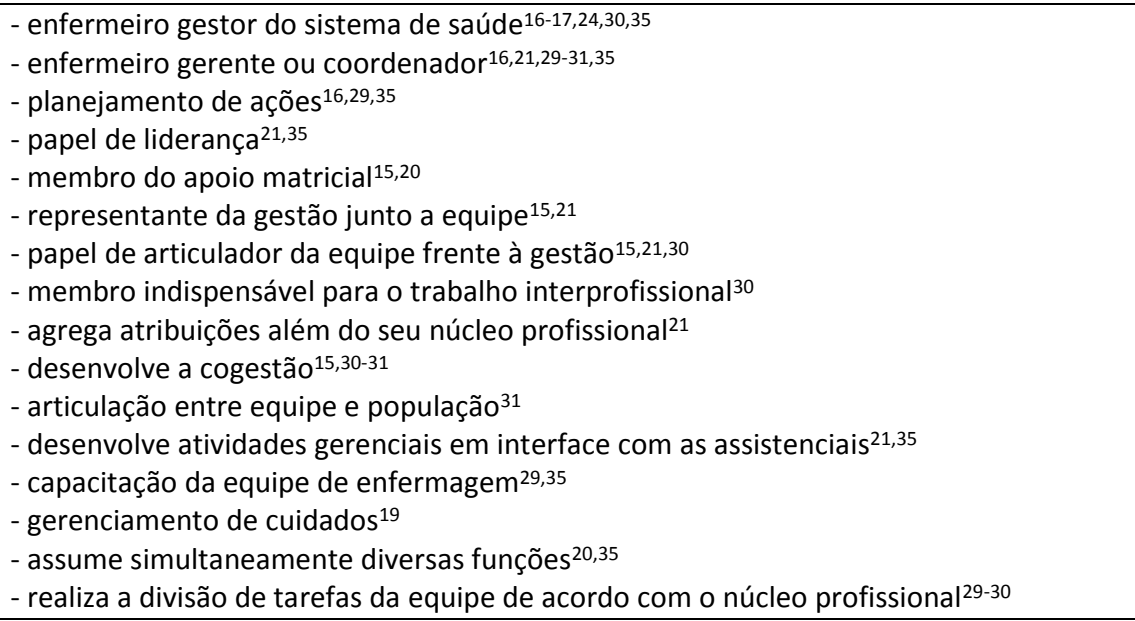 \\
\hline $\begin{array}{l}\text { Sistêmica } \\
/ 19,27,29,30,32,33 \\
36,37,38\end{array}$ & $\begin{array}{l}\text { - integralidade da assistência } 29-30 \\
\text { - troca de conhecimentos entre referência e contrarreferência } 29-30 \\
\text { - sistema de comunicação entre os serviços de saúde }{ }^{30,36} \\
\text { - transição do cuidado }{ }^{33} \\
\text { - processo de notificação } \\
\text { - rede de cuidados colaborativos } \\
\text { - contexto do sistema de saúde }{ }^{36} \\
\text { - uso adequado de recursos }{ }^{19} \\
\text { - integração dos serviços e reorientação do foco do atendimento }{ }^{37} \\
\text { - presença de órgãos regulatórios }{ }^{27}\end{array}$ \\
\hline $\begin{array}{l}\text { Societária } \\
/ 22,23,32 \text {. }\end{array}$ & $\begin{array}{l}\text { - políticas de saúde }{ }^{32} \\
\text { - mudanças no modelo de atenção } \\
\text { - comissão de saúde }\end{array}$ \\
\hline
\end{tabular}

FIGURA 3: Síntese dos achados nos estudos que integraram a amostra para o período de 2012 a 2018. Florianópolis, Santa Catarina, Brasil, 2019.

\section{DISCUSSÃO}

\section{Dimensões da Gestão do Cuidado presentes na prática do Enfermeiro na APS}

O recorte temporal não possibilita caracterizar um padrão de crescimento das publicações, embora seja possível inferir um aumento das produções em 2016. Uma predominância de estudos qualitativos pode estar relacionada à importância da compreensão da dimensão subjetiva da gestão do cuidado. Porém, pode-se acenar para a necessidade do investimento em estudos com outras abordagens metodológicas que deem conta de questões abrangendo diferentes dimensões da gestão do cuidado, em especial a individual, familiar, e societária, e que avaliem objetivamente os resultados obtidos a partir da atuação dos enfermeiros na gestão do cuidado. 
A predominância de estudos nacionais pode estar associada à adoção de um marco histórico brasileiro para a seleção dos artigos, ou seja, a $\mathrm{PNAB}^{13}$. Além disso, a nomenclatura "gestão do cuidado" é utilizada principalmente no cenário nacional. Em outros países, utiliza-se, por exemplo, "gestão da clínica" para se referir à práticas gerencias visando a uma assistência segura, eficiente, equitativa e humanizada ${ }^{39}$.

Com base na análise dos estudos selecionados, identificou-se o destaque na prática do enfermeiro no contexto da APS para a dimensão organizacional, seguida das dimensões profissional e sistêmica, respectivamente. A análise em profundidade dos artigos possibilitou identificar características que puderam ser relacionadas a mais de uma dimensão da gestão do cuidado em dez estudos ${ }^{19-20,24-25,27,29-32,35}$. O protagonismo do enfermeiro na equipe de saúde sobressaiuse nos estudos, evidenciando a multiplicidade de atividades que este profissional assume simultaneamente em relação à condução da equipe e a organização do trabalho.

O foco dos artigos concentra-se no trabalho gerencial do enfermeiro na APS, deixando para segundo plano a sua influência na autonomia dos pacientes e seu mundo da vida, no financiamento e nas políticas sociais. A presença de poucos estudos envolvendo as dimensões individual e familiar pode ter contribuído para a escassez de resultados relativos a essa área, pois apenas quatro estudos envolveram pacientes. Do mesmo modo, na dimensão societária somente dois estudos incluíram especificamente gestores e gerentes.

Na dimensão individual, os estudos ilustram o cuidar de si por meio de sentir-se responsável pelo autocuidado e ser capaz de realizar atividades diárias. $O$ desenvolvimento de habilidades para o autocuidado é evidenciado pelo auto manejo de doenças crônicas e escolha de estilos de vida ${ }^{18,25,32}$, e o enfermeiro realiza o gerenciamento de pacientes com doenças de longo prazo ${ }^{18}$.

Na dimensão familiar, os estudos apontam o suporte de parentes e amigos como fatores que contribuem para a adesão de pessoas com doenças crônicas a tratamentos e a reabilitação da saúde ${ }^{25,30}$. A família desempenha papel relevante no cuidado aos usuários, mas também precisa de educação e apoio, e nesse sentido, a terapia de família integra o tratamento ${ }^{19,32}$.

Em relação à dimensão profissional, a prática assistencial do enfermeiro por meio da consulta e do processo de enfermagem contribui para a integralidade no cuidado ${ }^{34}$, ainda que alguns gestores apontem o processo de enfermagem como uma prática que dificulta a assistência devido a sua complexidade e especificidade ${ }^{24}$.

A competência técnica é identificada pela prática baseada em evidências e busca pela segurança e qualidade do cuidado, sendo exemplificada pelo desenvolvimento de plano de cuidados ${ }^{19,34}$ e procedimentos padronizados ${ }^{27}$. Apesar disso, há fatores que interferem na habilidade profissional do enfermeiro, como: não ter recebido ou buscado formação para desenvolver competências necessárias a determinado tipo de atendimento ${ }^{26}$, carga de trabalho excessiva e fadiga $^{28}$. Assim, pontua-se a necessidade de suprir lacunas do ensino formal por meio de capacitação e cursos de especialização visando ao desenvolvimento de competências profissionais ${ }^{27,35}$.

Para além do preparo técnico, a dimensão profissional contempla vínculos e aspectos éticos. O vínculo estabelecese na relação entre enfermeiros e pacientes devido à proximidade entre eles, e o aspecto ético reside na preocupação com a prestação dos cuidados ${ }^{27}$. Na relação entre enfermeiros e médicos, há dificuldade em discutir determinados temas quando as opiniões são discordantes ${ }^{28}$. A relação do enfermeiro com a equipe de saúde é permeada de obstáculos como a incompreensão sobre o seu papel e condutas impróprias de alguns membros da equipe, como postura ética inadequada, falta de comprometimento e desmotivação ${ }^{20,31,35}$.

$\mathrm{Na}$ dimensão organizacional, os estudos discutem os papeis formais e informais que permeiam o trabalho gerencial do enfermeiro. Os papeis formais referem-se à atuação do enfermeiro como gestor do sistema de saúde ${ }^{16-}$ 17,24,30,35, como gerente ou coordenador responsável pela condução da unidade de saúde ${ }^{16,21,29-31,35}$, por meio de ações que envolvem planejamento, controle e avaliação dos processos de trabalho ${ }^{16}$. Independente do exercício formal de um cargo gerencial, o trabalho do enfermeiro envolve a prática da liderança frente à equipe de saúde, gestão de conflitos, delegação de $\operatorname{tarefas}^{21,35}$; membro do apoio matricial, acompanhando os processos de trabalho de cada setor $^{15,20}$; representante da gestão junto a equipe ${ }^{15,21}$; e articulador da equipe frente à gestão, atuando junto ao usuários e à equipe multiprofissional ${ }^{15,21,30}$.

Dessa forma, o enfermeiro é visto pela equipe como membro indispensável para o trabalho interprofissional ${ }^{30}$, agregando atribuições para além do seu núcleo profissional ${ }^{21}$. A atuação do enfermeiro também envolve a construção de espaços de cogestão ${ }^{15,30-31}$ que facilitam o trabalho em equipe, pois possibilitam a articulação entre os membros da equipe e a população na implementação das ações ${ }^{31}$.

As práticas gerenciais estão inseridas na formação e atuação do enfermeiro, o que contribui para que ele seja o profissional mais ligado à coordenação do serviço de saúde ${ }^{21,31,35}$. Contudo, estas práticas requerem habilidades pouco desenvolvidas durante a formação, sugerindo que esta complementação seja oferecida pela gestão em saúde ${ }^{21}$.

Os enfermeiros desempenham atividades gerenciais em interface com as assistenciais ${ }^{21,35}$. Essa atuação integrada requer assumir simultaneamente funções relacionadas à equipe, ao serviço, à assistência e ao planejamento de ações. 
Os enfermeiros ainda são responsáveis pela capacitação da equipe de enfermagem para questões assistenciais e administrativas ${ }^{29,35}$ e desenvolvem o gerenciamento de cuidados a pacientes crônicos ${ }^{19}$. 0 enfermeiro obtém o reconhecimento da equipe em virtude da diversidade de atividades que executa ${ }^{20,35}$.

As reuniões de equipe possibilitam que o enfermeiro conduza o trabalho de modo conjunto com a equipe multiprofissional e desenvolva novos processos voltados à integralidade do cuidado. Contudo, estudos apontam a carência na sistematização de ações de cuidado exclusivas dos enfermeiros, resultando em uma gestão fragmentada e isolada ${ }^{17,30}$.

A divisão técnica do trabalho, também contemplada na dimensão organizacional, permite observar a ênfase atribuída à divisão de tarefas pela equipe de acordo com o núcleo profissional. O trabalho é discutido coletivamente, mas se restringe à decisão de quem faz o que, fragmentando a coordenação e a continuidade do cuidado ${ }^{29-30}$. Apesar disso, a enfermagem assume a maior parte das atividades, absorvendo as atividades de outros profissionais e deixando de reconhecer a necessidade da divisão de tarefas. Além disso, uma postura centralizadora do enfermeiro, como nas atividades de comunicação com outros setores, gera estresse ocupacional e limita os técnicos e auxiliares a condutas assistenciais $^{29}$.

A dimensão sistêmica da gestão do cuidado remete às linhas de cuidado e ao financiamento. Nessa direção, a integralidade da assistência pode ser obtida pela equipe de saúde por meio da troca de conhecimentos entre referência e contrarreferência entre os diferentes pontos da rede promovendo uma prática interdisciplinar e resolutiva ${ }^{29-30}$. Desse modo, um sistema de comunicação eficaz entre os serviços de saúde pode ser uma opção para a resolução dos obstáculos na rede de atenção ${ }^{30,36}$, em especial no momento de transição do cuidado entre APS e hospital ${ }^{33}$. A adequada comunicação é imprescindível no processo de notificação, assim como para superar dificuldades na rede assistencial relacionadas as ações intersetoriais e capacitação profissional ${ }^{38}$.

A rede de cuidados colaborativos possibilita a integração entre os serviços de atenção primária e saúde mental, focando na liderança, na equipe, no desenho do programa, nos desafios relacionados à cultura organizacional e clínica, na melhoria da qualidade do cuidado, na tecnologia de informação em saúde, e no financiamento ${ }^{32}$. A compreensão do contexto do sistema de saúde permite que os enfermeiros direcionem a resolutividade do cuidado considerando a relação entre os recursos, a estrutura e o modelo de gestão ${ }^{36}$. O conhecimento sobre financiamento e uso adequado de recursos oportuniza a diminuição de custos, de modo que sejam suficientes para compensar o custo com recursos e pessoal adicional ${ }^{19}$.

Nesse sentido, aponta-se um estudo acerca da implementação de um sistema de saúde ocular o qual resultou na significativa ampliação do número de exames oftalmológicos oferecidos por enfermeiros treinados na APS, integrando e reorientando o foco do atendimento nos hospitais e centros de saúde ${ }^{37}$. Logo, as políticas de enfermagem e saúde são necessárias para organizar um quadro institucional operante, e os sistemas educacionais favorecem o desenvolvimento das competências de enfermagem, além de contribuir com os órgãos regulatórios ${ }^{27}$.

Na dimensão societária da gestão do cuidado, as políticas de saúde garantem a sustentabilidade de programas, diminuem as barreiras para o desenvolvimento de estratégias inovadoras para o reembolso dos serviços, valorizando a gestão e coordenação do cuidado ${ }^{32}$. Mudanças no modelo de atenção requerem a revisão de atitudes profissionais e ampliação dos saberes, olhares, relações de trabalho e poder entre a equipe de saúde, com vistas a superação de posturas instituídas que não condizem com as diretrizes preconizadas pelas políticas públicas de saúde ${ }^{23}$. A comissão de saúde mostra-se um exemplo de estratégia de interlocução para a busca de espaços democráticos na APS, promovendo controle social e educação permanente em saúde ${ }^{22}$.

\section{Limitações do estudo}

Como limitações, considera-se que os descritores adotados podem ter contribuído para a identificação de poucos achados relacionados as dimensões individual, familiar e societária da gestão do cuidado. Além disso, o recorte temporal em consonância com o ano em que a APS é reorganizada no Brasil por meio de sua Política, sem considerar essa temporalidade em outros países, pode ter influenciado na concentração de estudos nacionais.

\section{CONCLUSÃO}

Constatou-se que todas as dimensões da gestão do cuidado estão presentes na prática do enfermeiro na APS, com destaque para as dimensões organizacional, profissional e sistêmica. Na dimensão organizacional, reforça-se a importância do papel gerencial do enfermeiro enquanto gestor, gerente ou coordenador, por meio do exercício da liderança, planejamento de ações e desenvolvimento de atividades gerenciais integradas à assistência.

A dimensão profissional está presente na prática do enfermeiro especialmente em atividades como a consulta e o processo de enfermagem, a prática baseada em evidências, a realização do plano de cuidados, no vínculo com os pacientes, e na relação com a equipe de saúde. A dimensão sistêmica pontua a integralidade da assistência, requer a troca de conhecimentos entre referência e contrarreferência, e o sistema de comunicação entre os serviços de saúde. 
As dimensões individual, familiar e societária aparecem menos nos estudos, mas também estão presentes na prática do enfermeiro na APS, remetem ao auto manejo das pessoas frente as situações crônicas, ao suporte oferecido por parentes e amigos, e as mudanças no modelo de atenção, respectivamente.

Os estudos apontam a necessidade do desenvolvimento contínuo de competências pelo enfermeiro durante a sua trajetória profissional, sendo necessário apreender novos, outros conhecimentos, incluindo os que ultrapassam seu núcleo de saber, o que se constitui como oportunidade de melhoria para a sua atuação no contexto da APS, bem como para o seu posicionamento frente a opiniões discordantes, contribuindo na relação entre a equipe, gestão e população.

Para a continuidade e o fortalecimento das dimensões da gestão do cuidado na prática do enfermeiro na APS, sugere-se inovações e adoção de diferentes abordagens metodológicas em pesquisas futuras, uma vez que a maioria dos estudos analisados foram qualitativos. Além disso, é importante abordar outras questões, tais como: atuação do enfermeiro na promoção da autonomia do paciente, no envolvimento da família e amigos no cuidado, efeitos da implementação de sistemas de comunicação, financiamento e políticas sociais.

\section{REFERÊNCIAS}

1. Soder RM, Santos LE, Oliveira IC, Silva LAA, Peiter CC, Santos JLG. Healthcare management practices in primary care. Rev. Cubana Enferm. [Internet]. 2020 [cited 2020 Oct 20]; 36(1):e2815. Available from:

http://www.revenfermeria.sld.cu/index.php/enf/article/view/2815.

2. Souza GRM, Cazola LHO, Pícoli, RP. The work of primary health care nurses in oncology care: an Integrative review. Cogitare Enferm [Internet]. 2018 [cited 2020 Oct 20]; 23(4):e58152. DOI: http://dx.doi.org/10.5380/ce.v23i4.58152.

3. Dias FA, Gama ZAS, Tavares DMS. Primary health care to the elderly: a conceptual model of nursing. Cogitare Enferm. [Internet]. 2017 [cited 2020 Oct 20]; 22(3):e53224. DOI: http://dx.doi.org/10.5380/ce.v22i3.53224.

4. Mororó DDS, Enders BC, Lira ALBC, Silva CMB, Menezes RMP. Concept analysis of nursing care management in the hospital context. Acta Paul. Enferm. [Internet]. 2017 [cited 2020 Oct 20]; 30(3):323-32. DOI: https://doi.org/10.1590/19820194201700043.

5. Lucca TRS, Vannuchi MTO, Garanhani ML, Carvalho BG, Pissinati PSC. The meaning of care management attributed by nursing faculty members from the viewpoint of complex thinking. Rev. Gaúch. Enferm. [Internet]. 2016 [cited 2020 Oct 20]; 37(3):e61097. DOI: https://doi.org/10.1590/1983-1447.2016.03.61097.

6. Santos JLG, Pestana AL, Guerrero P, Meirelles BSH, Erdmann AL. Nurses' practices in the nursing and health care management: integrative review. Rev. Bras. Enferm. [Internet]. 2013 [cited 2020 Oct 20]; 66(2):257-63. DOI: https://doi.org/10.1590/S003471672013000200016.

7. Andrade SR, Schmitt MD, Schittler ML, Ferreira A, Ruoff AB, Piccoli T. Configuration of the management of nursing care in Brazil: a documentary analysis. Enferm. Foco [Internet]. 2019 [cited 2020 Oct 20]; 10(1):127-33. DOI: https://doi.org/10.21675/2357707X.2019.v10.n1.1926.

8. Peduzzi M, Leonello VM, Ciampone MHT. Trabalho em equipe e prática colaborativa. In: Kurcgant P (coord.). Gerenciamento em enfermagem. Rio de Janeiro: Guanabara Koogan; 2016. p. 103-13.

9. Siewert JS, Rodrigues DB, Malfussi LBH, Andrade SR, Erdmann AL. Management of integral care in nursing: reflections under the perspective of complex thinking. Reme, Rev. Min. Enferm. [Internet]. 2017 [cited 2020 Oct 20]; 21:e1047. DOI: http://www.dx.doi.org/10.5935/1415-2762.20170057.

10. Cecílio LCO. Theoretical and conceptual notes on evaluative processes taking the multiple dimensions of healthcare management into account. Interface (Botucatu) [Internet]. 2011 [cited 2020 Oct 20]; 15(37):589-99. DOI: https://doi.org/10.1590/S1414-32832011000200021.

11. Treviso P, Peres SC, Silva AD, Santos AA. Nursing skills in care management. Rev. Adm. Saúde [Internet]. 2017 [cited 2020 Oct 20]; 17(69). DOI: http://dx.doi.org/10.23973/ras.69.59.

12. Ganong LH. Lawrence H. Integrative review of nursing research. Research Nursing in Health [Internet]. 1987 [cited 2020 Oct 20]; 10(1):1-11. DOI: https://doi.org/10.1002/nur.4770100103.

13. Ministério da Saúde (BR), Gabinete do Ministro. Portaria no 2.488, de 21 de outubro de 2011. Aprova a Política Nacional de Atenção Básica, estabelecendo a revisão de diretrizes e normas para a organização da Atenção Básica, para a Estratégia Saúde da Família (ESF) e o Programa de Agentes Comunitários de Saúde (PACS). Brasília, DF: Ministério da Saúde, 2011. [cited 2020 Oct 20]. Available from: http://bvsms.saude.gov.br/bvs/saudelegis/gm/2011/prt2488_21_10_2011.html.

14. Bardin L. Análise de Conteúdo. Trad. de Luís Antero Reto, Augusto Pinheiro. São Paulo: Edições 70; 2011.

15. Barrêto AJR, Romera AA, Oliveira AAV, Sá LD, Almeida SA, Nogueira JA. The matrix supporter's work in the family health strategy. Rev Rene [Internet]. 2012 [cited 2020 Oct 20]; 13(1):166-77. Available from: http://www.repositorio.ufc.br/bitstream/riufc/12868/1/2012_art_ajrbarreto.pdf.

16. Neto FRGX, Coelho SJJ. Analysis of the workíng process of family health strategy territory managers. Rev. Gerenc. Polit. Salud [Internet]. 2012 [cited 2020 Oct 20]; 11(22):76-91. Available from: http://www.scielo.org.co/scielo.php?script=sci_arttext\&pid=S1657-70272012000100007\&lng=en\&tIng=en\&gathStatlcon=true

17. Costa RF, Queiroz MVO, Zeitoune RCG. Care to adolescents: contributing to nursing. Rev. Enferm. UERJ [Internet]. 2012 [cited 2020 Oct 20]; 20(2):197-202. Available from: https://www.epublicacoes.uerj.br/index.php/enfermagemuerj/article/view/4045/2809. 
18. Kennedy A, Bower P, Reeves D, Blakeman T, Bowen R, Chew-Graham C, et al. Implementation of self-management support for long term conditions in routine primary care settings: cluster randomised controlled trial. BMJ [Internet]. 2013 [cited 2020 Oct 20]; 346:f2882. DOI: https://doi.org/10.1136/bmj.f2882.

19. Marsteller JA, Hsu YJ, Wen W, Wolff J, Frick K, Reider L, et al. Effects of guided care on providers' satisfaction with care: a threeyear matched-pair cluster-randomized trial. Popul. Health Manag. [Internet]. 2013 [cited 2020 Oct 20]; 16(5):317-25. DOI: https://doi.org/10.1089/pop.2012.0091.

20. Romera AA, Barrêto AJR, Sá LD, Almeida AS, Nogueira JA, Sá CMCP. Matrix support work: difficulties in the scope of basic healthcare. Rev. Gaúch. Enferm. [Internet]. 2014 [cited 2020 Oct 20]; 35(1):140-47. DOI: https://doi.org/10.1590/19831447.2014.01.40141.

21. Melo RC, Machado ME. Coordination of family healthcare units done by nurses: challenges and potential. Rev. Gaúch. Enferm. [Internet]. 2013 [cited 2020 Oct 20]; 34(4):61-7. DOI: https://doi.org/10.1590/S1983-14472013000400008.

22. Ramos LS, Beck CLC, Freitas NQ, Dissen CM, Fernandes MNS, Colomé ICS. Mental health commission: strategies for creation of interface in primary care. Rev. Enferm. UERJ [Internet]. 2013 [cited 2020 Oct 20]; 21(esp1):581-6. Available from: https://www.e-publicacoes.uerj.br/index.php/enfermagemuerj/article/view/10002.

23. Silva JS, Fortuna CM, Pereira MJB, Matumoto S, Santana FR, Marciana FM, et al. Supervision of Community Health Agents in the Family Health Strategy: the perspective of nurses. Rev. Esc. Enferm. USP [Internet]. 2014 [cited 2020 Oct 20]; 48(5):899-906. Available from: https://www.researchgate.net/publication/273957042_Supervision_of_Community_Health_Agents_in_the_Family_Health_Str ategy_the_perspective_of_nurses.

24. Diniz IA, Cavalcante RB, Otoni A, Mata LRF. Perception of primary healthcare management nurses on the nursing process. Rev. Bras. Enferm. [Internet]. 2015 [cited 2020 Oct 20]; 68(2):206-13. DOI: http://dx.doi.org/10.1590/0034-7167.2015680204i.

25. Hirsikangas S, Kanste O, Korpelainen J, Kynga H. Adherence to health regimens among frequent attenders of Finnish healthcare. International Journal of Circumpolar Health [Internet]. 2016 [cited 2020 Oct 20]; 75:30726. DOI: http://dx.doi.org/10.3402/ijch.v75.30726.

26. Mori EC, Naghettini AV. Medical training and nurses of Family Health strategy on worker health aspect. Rev. Esc. Enferm. USP [Internet], 2016 [cited 2020 Oct 20]; 50(spe):25-31. DOI: https://doi.org/10.1590/S0080-623420160000300004.

27. Ausserhofer D, Rakic S, Novo A, Dropic E, Fisekovic E, Sredic A, et al. Improving the safety and quality of nursing care through standardized operating procedures in Bosnia and Herzegovina. Int. nurs. rev. [Internet]. 2016 [cited 2020 Oct 20]; 63(2):208-17. DOI: https://doi.org/10.1111/inr.12237.

28. Gabrani JC, Knibb W, Petrela E, Hoxha A, Gabrani A. Provider perspectives on safety in primary care in Albania. J. Nurs. Scholars. [Internet]. 2016 [cited 2020 Oct 20]; 48(6):552-60. DOI: https://doi.org/10.1111/jnu.12236.

29. Almeida JB, Ponce MAZ, Wysocki AD, Santos MLSG, Vendramini SHF. The coordination of assistance in tuberculosis control in the nursing team's view. Rev. enferm. UFPE on line. [Internet], 2016 [cited 2020 Oct 20]; 10(Supl. 6):4727-34. Available from: https://periodicos.ufpe.br/revistas/revistaenfermagem/article/download/11250/12865.

30. Bandeira D, Weiller TH, Silveira TF, Damaceno AN, El Hodali NF. Attention users management with dependence of accident after-effects for care vascular brain. Rev. APS [Internet]. 2016 [cited 2020 Oct 20]; 19(4):575-81. Available from: https://periodicos.ufjf.br/index.php/aps/article/view/15684.

31. Borges FA, Ogata MN, Feliciano AB, Fortuna CM. Management in health: the workers look of a rural family health. Ciênc. Cuid. Saúde [Internet]. 2016 [cited 2020 Oct 20]; 15(3):466-73. DOI: https://doi.org/10.4025/cienccuidsaude.v15i3.31531.

32. Price-Haywood EG, Dunn-Lombard D, Harden-Barrios J, Lefante JJ. Collaborative depression care in a safety net medical home: facilitators and barriers to quality improvement. Popul. Health Manag. [Internet]. 2016 [cited 2020 Oct 20]; 19(1):46-55. DOI: https://doi.org/10.1089/pop.2015.0016.

33. Coelho APC, Larocca LM, Chaves MMN, Felix JVC, Bernardino E, Alessi SM. Healthcare management of tuberculosis: integrating a teaching hospital into primary health care. Texto Contexto Enferm. [Internet]. 2016 [cited 2020 Oct 20]; 25(2):e0970015. DOI: http://dx.doi.org/10.1590/0104-07072016000970015.

34. Castro-Sampaio F, Oliveira PP, Mata LRF, Moraes JT, Fonseca DF, Vieira VAS. Profile of nursing diagnoses in people with hypertension and diabetes. Invest. educ. enferm. [Internet]. 2017 [cited 2020 Oct 20]; 35(2):139-53. DOI: http://dx.doi.org/10.17533/udea.iee.v35n2a03.

35. Celedônio RM, Fé MCM, Mendes AHL, Mendes AHL, Chaves TLF. Management of work in basic health units. Rev enferm UFPE on line. [Internet]. 2017 [cited 2020 Oct 20]; 11(Suppl. 1):341-50. Available from: https://periodicos.ufpe.br/revistas/revistaenfermagem/article/view/11914.

36. Soder R, Oliveira IC, Silva LAA, Santos JLG, Peiter CC, Erdmann AL. Challenges for the care management in primary health care: perspective of the nursing team. Enferm. Foco [Internet]. 2018 [cited 2020 Oct 20]; 9(3):76-80. DOI: https://doi.org/10.21675/2357-707X.2018.v9.n3.1496.

37. Yip JL, Bright T, Ford S, Mathenge W, Faal H. Process evaluation of a National Primary Eye Care Programme in Rwanda. BMC Health Serv. Res. [Internet]. 2018 [cited 2020 Oct 20]; 18:950. DOI: https://doi.org/10.1186/s12913-018-3718-1.

38. Egry EY, Apostolico MR, Morais TCP. Reporting child violence, health care flows and work process of primary health care professionals. Ciênc. Saúde Colet. [Internet]. 2018 [cited 2020 Oct 20]; 23(1):83:92. DOI: https://doi.org/10.1590/141381232018231.22062017.

39. Mendes EV. As redes de atenção à saúde [Internet]. Brasília: Organização Pan-Americana da Saúde. 2011 [cited 2020 Oct 20]. Available from: https://www.paho.org/bra/index.php?option=com_docman\&view=download\&category_slug=servicos-saude095\&alias=1402-as-redes-atencao-a-saude-2a-edicao- $2 \&$ Itemid $=965$. 